\section{The psychiatric impact of COVID-19 on healthcare workers}

CPD

Sonja Cabarkapa, Joel A King, Chee $\mathrm{H} \mathrm{Ng}$

\section{Background}

Healthcare workers (HCWs) are a vulnerable population who have been exposed to high work-related stress during the COVID-19 pandemic because of the high risk of infection and excessive workloads. HCWs are at greater risk of mental illness, particularly sleep disturbances, post-trauma stress syndromes, depression and anxiety.

\section{Objective}

The aim of this article is to highlight the psychiatric impact of the COVID-19 pandemic on frontline HCWs, the need for screening and early diagnosis by general practitioners (GPs), and the appropriate psychosocial strategies and treatments to address this.

\section{Discussion}

Opportunistic screening for mental health issues among HCWs is especially important during the current pandemic. Various tools and strategies can be used for efficient assessment and treatment of the common mental health issues HCWs are likely to face.

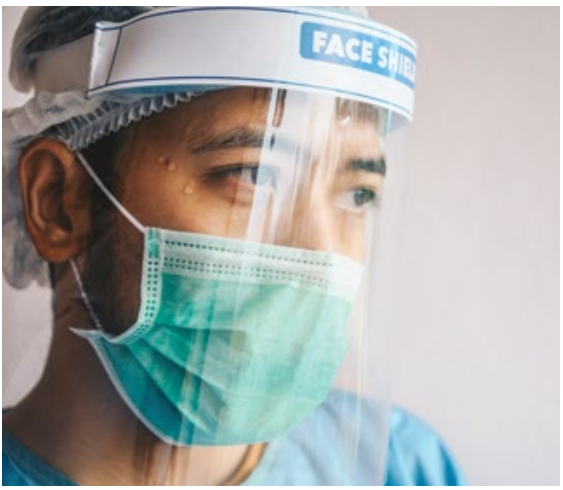

HEALTHCARE WORKERS (HCWs) are vulnerable to developing trauma or stress-related disorders in addition to other mental health conditions during large-scale disease outbreaks. If not adequately addressed, the negative impact on their mental health may lead to long-term psychological consequences. In general, HCWs have a higher risk of mental health problems - including a greater risk of suicide, suicidal ideation and self-harm behaviours - than the general population. ${ }^{1}$ During a pandemic, they are particularly vulnerable to mental health issues because of the high risk of infection, increased work stress and fear of spreading the virus to their families. ${ }^{2}$ External factors, such as shift work and high-pressure work environments, amplify the risks of burn-out and depression. The concept of work-life balance has long been a method of sustaining wellbeing and mitigating these risks. ${ }^{3}$ However, high work demands, fear and various social restrictions during the pandemic have impeded common ways to maintain work-life balance.

The fear of becoming infected during a large-scale disease outbreak is among the most common psychological challenges faced. ${ }^{4}$ Similarly, the fear of uncertainty is significant and attributed to the direct and economic effects of the pandemic.
Stigma, especially in the context of social isolation and being in quarantine, has been reported as a prominent psychological stressor in previous outbreaks. ${ }^{5-7}$ While the media can play a role in amplifying uncertainty, ${ }^{8}$ it can also be used as a psychological resource, and recent findings suggest that it can enhance access to psychological materials. ${ }^{9}$

During the COVID-19 pandemic, HCWs have been exposed to high infection risks, mortality, moral dilemmas, excessive workloads and ongoing uncertainty about the pandemic wave. The entire experience can be traumatising, with several studies showing an increased risk of acquiring a trauma or stress-related disorder. ${ }^{10}$ General practitioners (GPs) play a pivotal role as a first point of contact for HCWs as well as the broader community experiencing mental health challenges. Thus, GPs face several critical stressors including the increased prevalence of mental health issues in patients, difficulties accessing adequate supplies of personal protective equipment (PPE) and adverse financial impacts, initially exacerbated by the Federal Government's restrictions on mandatorily bulk-billed telehealth consultations. As frontline HCWs, it is essential that GPs have their own doctor to assist in maintaining their health. 


\section{Clinical features}

The impact of COVID-19 on the mental wellbeing of HCWs has been profound, and findings from international studies can help prepare the Australian health sector for the potential repercussions. Studies have reported a range of psychiatric morbidities in frontline HCWs during the COVID-19 pandemic. These are predominantly post-traumatic stress syndromes, depression and anxiety, ${ }^{9-18}$ but also include insomnia, psychological distress, obsessive-compulsive symptoms and somatisation. ${ }^{18}$ More than $70 \%$ of a group of $>1200 \mathrm{HCWs}$ reported psychological distress with high rates of depression, anxiety and insomnia. ${ }^{19}$ Symptoms of insomnia have been reported by approximately $36 \%$ of HCWs. ${ }^{20}$ Anxiety has been shown to be a significant factor affecting nurse ${ }^{14}$ and staff with direct contact with infected patients. ${ }^{17}$ Long work hours were also found to increase stress levels among nurses, ${ }^{14}$ and approximately two-thirds of HCWs working on the frontline reported moderate-to-high levels of stress. ${ }^{21}$ In one cohort of 4000 HCWs, approximate rates of mental health issues were $15 \%$ for depression, 25\% for anxiety and 30\% for acute stress symptoms. ${ }^{22}$ The burden of adhering to strict protective measures was also highlighted as a contributor to levels of distress. ${ }^{20} \mathrm{HCWs}$ who viewed the protection as insufficient rated higher levels of depression, anxiety and acute stress than those who perceived it to be adequate. ${ }^{16}$

\section{Screening and diagnosis}

Mental health issues are often under-recognised among HCWs and can be easily missed. It is critical that any HCW, regardless of whether they are exposed to patients with COVID-19, is asked about their mental health. This includes administrative, allied health and non-clinical staff who work in medical settings. Even closer attention needs to be given to those who are frontline workers. Quick screening tools such as those listed in Table 1 could be useful and practical. Although the use of telehealth has obvious benefits, ${ }^{23,24}$ it can impede the value of gaining an adequate in-person mental state examination, which remains paramount to the psychiatric interview. Therefore, video calls are preferred and can offer even greater insights as the patient is often calling from home, allowing inferences about their demeanour and environment to be made.

Findings from previous outbreaks show variable coping strategies between contrasting sociocultural settings and differences existing among doctors, nurses and other HCWs. In Australia, it has been reported that $40 \%$ of individuals use alcohol to cope with stress, ${ }^{25}$ and this is likely to increase during a pandemic. ${ }^{26-28}$ Simple screening questionnaires such as the CAGE questionnaire ${ }^{29}$ can be used to screen and monitor for alcohol dependence.

\section{General treatment strategies} The World Health Organization (WHO) recommends the timely provision of psychological first aid (PFA) for HCWs in need of support. ${ }^{30}$ PFA aims to reduce initial distress, address basic needs and practical supports, and promote adaptive coping and engagement with existing supports. Other guidelines also relay key messages ${ }^{31}$ to remind HCWs to prioritise their mental health. Lifestyle factors, work and psychosocial interventions remain at the forefront of management approaches. These key approaches to delivering interventions to address mental health issues in HCWs are outlined in the following sections of this article.

\section{Lifestyle/wellbeing strategies}

Feeling stressed is a normal reaction, not a failure. Remind HCWs that there is no health without mental health. Encourage basic needs, including healthy food, exercise, sleep, rest and recreation.

Enquire about adequate sunlight exposure, particularly with shift-workers. Probe for financial difficulties and refer to Centrelink or social workers where appropriate. Highlight the need for ongoing connection with family and friends. Advise patients to limit excessive and repetitive media use and suggest scheduled media-free days since recent evidence shows a strong association between mental health problems and frequent social media use during this pandemic. ${ }^{32}$

Adaptations for strategies are needed for people in isolation, and it is important to enquire about adequate personal space. Suggest maintaining a tidy environment with minimal clutter, using home sports equipment where possible; if unavailable, towels and heavy objects can be used as makeshift mats and weights. Reminding patients that the isolation period is temporary and provides a significant purpose to protect others may be helpful. Encourage regular interactions with friends, family and colleagues through telephone, video conferences and email, with scheduled times. Advise patients to maintain a varied activity schedule as the brain seeks novelty.

Enquire about workplace supports such as mentors, debrief sessions and other avenues to address their concerns.

\section{Table 1. Screening for mental illnesses during COVID-19}

\begin{tabular}{ll}
\hline Screening tool & Description \\
\hline $\begin{array}{l}\text { Generalised Anxiety Disorder } \\
\text { 7-item (GAD-7) scale }\end{array}$ & $\begin{array}{l}\text { A seven-item self-report anxiety scale with good reliability. } \\
\text { Efficient for screening for GAD and assessing its severity. }{ }^{39}\end{array}$ \\
\hline PTSD Checklist for DSM-5 & $\begin{array}{l}\text { A widely-used self-report measure of PTSD symptoms } \\
\text { reflecting the DSM-5. Demonstrates strong reliability } \\
\text { (PCL-5) }\end{array}$ \\
\hline $\begin{array}{l}\text { and validity. }{ }^{40} \\
\text { (PHQ-9) }\end{array}$ & $\begin{array}{l}\text { A self-report instrument for diagnosing major depressive } \\
\text { disorder in primary care and assessing severity. Good } \\
\text { brevity and construct/criterion validity. }\end{array}$ \\
\hline
\end{tabular}

DSM-5, Diagnostic and Statistical Manual of Mental Disorders, 5th edition; GAD, generalised anxiety disorder; PTSD, post-traumatic stress disorder 
Promote using pre-existing healthy coping mechanisms and avoiding unhealthy coping strategies (eg tobacco, alcohol and other drugs). Monitor for early warning signs of common psychiatric disorders, such as persistently low mood or irritability, excessive anxiety, unexplained physical complaints or increasing use of alcohol/substances. Educate about sleep, and practise sleep hygiene as first-line management for insomnia. Furthermore, provide advice about safe alcohol use and harm minimisation techniques. Many of these lifestyle issues exist outside of the pandemic and remain significant factors in HCW wellbeing for long-term consideration.

\section{Work interventions}

HCWs should have adequate and timely COVID-19 information and training relevant to their workplace. HCWs can be supported in decisions related to taking leave, including sick and annual leave, without feeling guilty or irresponsible. Setting work-life balance becomes difficult as the timing of workplace hours can easily become ill defined when working from one's home, thereby altering sleep-wake cycles, levels of productivity and job satisfaction. If the HCW works from home, this should be in a separate space, with defined hours and preferably separate work clothes.

\section{Psychosocial interventions}

Emphasise the need for scheduling time with family, friends and hobbies. Adapt prior hobbies that are restricted to alternatives, including online versions. Encourage other activities of daily living, such as shopping, that can still be done safely with careful planning around COVID-19 precautions. Psychological treatments should aim to lessen stress levels, offer education to empower individuals and enhance workplace supports. Cognitive behavioural therapy (CBT) is a first-line treatment for many high-prevalence disorders, including mild-to-moderate depression and most anxiety disorders. Relaxation techniques such as deep breathing and progressive muscle relaxation are cost effective, relatively easy to learn and broadly applicable. Numerous phone applications, including the free Stanford CBT-i Coach, offer a range of narrated techniques that are easy to follow. General information for younger individuals can easily be accessed through different websites, such as the Black Dog Institute, Beyond Blue, ReachOut and Head to Health. Online CBT courses such as THIS WAY UP and moodgym may be useful, and some also include COVID-19-specific modules.

Interpersonal therapy can be useful in helping HCWs to manage role transitions and role disputes. Changes in family roles and the greater amount of time that families may be spending together in the home may require various forms of family therapy, many of which can be delivered via telehealth as well as face to face. Increasing rates of family violence have been recorded during the pandemic, ${ }^{33}$ and HCWs are not immune to this. Enhancing supportive relationships with families, partners and significant others is also a key way to aid HCWs.

\section{Pharmacotherapies}

Appropriate pharmacotherapies may need to be instituted for moderate depression and anxiety disorders. More time spent at home, combined with winter months, can lead to vitamin D deficiency-related depression and should be treated with instructions regarding sunlight and/or vitamin D supplementation, depending on severity. Furthermore, vitamin D deficiency is a recognised risk factor for complicated COVID-19 infections; ${ }^{34}$ however, testing using Medicare Benefits Schedule reimbursement is restricted to those with recognised risk factors. ${ }^{35}$

The use of short-term hypnotics should be considered, as insomnia has also been found to be significantly higher in frontline HCWs. ${ }^{15}$ If possible, avoid using benzodiazepines for sleep issues and anxiety problems, given their addictive potential. If they are used, they should be used only in the short term, in combination with psychosocial treatments, with a plan for cessation. Furthermore, selective serotonin reuptake inhibitors are first-line agents in the treatment of major depressive and most types of anxiety disorders. Starting at a low dose and scheduling regular reviews can enhance the compliance by minimising side effects and ensuring adherence. Referral to a psychiatrist is indicated if the condition is severe, complex or high risk.

\section{Other treatment issues}

The severity of the pandemic often varies across different geographical locations or even hospitals and work settings, highlighting the contextual aspects of the stress placed on HCWs. Those without a history of any psychiatric disorder may present with a new-onset depressive or anxiety disorder triggered by the pandemic. Furthermore, stress can also arise from COVID-19-related somatic symptoms including headache, fatigue, vascular and neurological symptoms. ${ }^{36}$

Treating a fellow HCW can be challenging for multiple reasons; ${ }^{37}$ however, the same ethical and professional boundaries should remain. Many personal and psychological factors may influence provision of care given to HCWs, and these can reflect the treating doctor's own anxieties and fears. The judicious use of psychotropics within the individual clinical picture needs to be carefully considered. It is critical for the health sector as a whole to have an enhanced awareness of staff mental health, including the extent and sources of stress among HCWs during disease outbreaks. ${ }^{4}$ Moreover, hospital support systems and occupational health policy should be designed to promote the psychological wellbeing of HCWs. ${ }^{38}$ Improving the understanding of the levels of and factors associated with fear among staff could be useful in planning for future outbreaks of infectious disease. ${ }^{13}$

\section{Conclusion}

The risks to the mental wellbeing of HCWs in the current pandemic are multifaceted. If unrecognised, they can lead to long-term psychological consequences. Psychiatric comorbidities can be triggered but can potentially be mitigated with appropriate measures. GPs play an important role in providing early interventions for HCWs who may experience adverse psychological outcomes. Through opportunistic screening 
for mental health disorders and timely consideration of psychosocial interventions and treatment in both psychological and pharmacological modalities, the burden on the mental health of Australia's healthcare workforce can be lessened.

\section{Key points}

- Heightened fear and increased stress levels, especially for frontline HCWs during the COVID-19 pandemic, can lead to a range of psychological adversities. The effects can be long lasting if unrecognised and thus warrant urgent attention.

- As frontline HCWs, it is essential that GPs have their own doctor to assist in maintaining their health.

- Psychosocial strategies are often useful, but appropriate therapies to address mental health problems need to be easily accessible.

- GPs play an important role in caring for HCWs through proactive screening for mental health disorders and offering early interventions and effective treatments.

\section{Resources}

Useful Australian resources include:

- Headspace, https://headspace.org.au

- Black Dog Institute, www.blackdoginstitute.org.au

- Beyond Blue, www.beyondblue.org.au

- ReachOut, https://au.reachout.com

- Head to Health, https://headtohealth. gov.au

- National Debt Helpline, https://ndh.org.au

Cognitive behavioural therapy courses can be found at:

- Mental Health Online (offered by Swinburne University), www.mentalhealthonline.org.au

- THIS WAY UP, https://thiswayup.org.au

- moodgym, http://moodgym.com.au

\section{Authors}

Sonja Cabarkapa BPharm (Hons), MD, Psychiatry Registrar, St Vincent's Health, Vic; Psychiatry Registrar, The Professorial Unit, The Melbourne Clinic, Vic
Joel A King, MBBS, MPsy, FRANZCP, AFRACMA, AFAMEE, Cert Child Adol Psych, Consultant Psychiatrist and Director of Psychiatry Training, The Professorial Unit, The Melbourne Clinic, Vic; Senior Lecturer, The University of Melbourne, Vic Chee H Ng MBBS, MMed (Psych), MD, FRANZCP, Healthscope Chair of Psychiatry, The Professorial Unit, The Melbourne Clinic, Vic; Professor of Psychiatry, The University of Melbourne, Vic; Director, WHO Collaborating Centre in Mental Health, St Vincent's Hospital, Vic. cng@unimelb.edu.au Competing interests: JAK reports an honorarium from Servier for a presentation, outside of the submitted work. CHN reports travel support and an honorarium from both Lundbeck and Pfizer for a presentation, as well as travel support and an honorarium for a consulting service from Janssen, outside of the submitted work.

Funding: None.

Provenance and peer review: Commissioned, externally peer reviewed.

\section{References}

1. Dutheil F, Aubert C, Pereira B, et al. Suicide among physicians and health-care workers: A systematic review and meta-analysis. PLoS One 2019;14(12):e0226361. doi: 10.1371/journal. pone.0226361.

2. Xiang YT, Yang Y, Li W, et al. Timely mental health care for the 2019 novel coronavirus outbreak is urgently needed. Lancet Psychiatry 2020;7(3):228-29. doi: 10.1016/S22150366(20)30046-8.

3. Schwartz SP, Adair KC, Bae J, et al. Work-life balance behaviours cluster in work settings and relate to burnout and safety culture: $A$ cross-sectional survey analysis. BMJ Qual Saf 2019;28(2):142-50. doi: 10.1136/bmjqs-2018007933.

4. Tam CW, Pang EP, Lam LC, Chiu HF. Severe acute respiratory syndrome (SARS) in Hong Kong in 2003: Stress and psychological impact among frontline healthcare workers. Psychol Med 2004;34(7):1197-204. doi: 10.1017/ s0033291704002247.

5. Maunder RG, Lancee WJ, Rourke S, et al. Factors associated with the psychological impact of severe acute respiratory syndrome on nurses and other hospital workers in Toronto. Psychosom Med 2004;66(6):938-42. doi: 10.1097/01. psy.0000145673.84698.18.

6. Khee KS, Lee LB, Chai OT, Loong CK, Ming CW, Kheng TH. The psychological impact of SARS on health care providers. Crit Care Shock 2004;7:100-06.

7. Koh D, Lim MK, Chia SE, et al. Risk perception and impact of severe acute respiratory syndrome (SARS) on work and personal lives of healthcare workers in Singapore: What can we learn? Med Care 2005:43(7):676-82. doi: 10.1097/01. mlr.0000167181.36730.cc.

8. Shih FJ, Gau ML, Kao CC, et al. Dying and caring on the edge: Taiwan's surviving nurses' reflections on taking care of patients with severe acute respiratory syndrome. Appl Nurs Res 2007;20(4):171-80. doi: 10.1016/j. apnr.2006.08.007.

9. Kang L, Ma S, Chen M, et al. Impact on mental health and perceptions of psychological care among medical and nursing staff in Wuhan during the 2019 novel coronavirus disease outbreak: A cross-sectional study. Brain Behav Immun 2020;87:11-17. doi: 10.1016/j.bbi.2020.03.028

10. Cabarkapa S, Nadjidai SE, Murgier J, Ng CH. The psychological impact of COVID-19 and other viral epidemics on frontline healthcare workers and ways to address it: A rapid systematic review. Brain Behav Immun Health 2020;8:100144. doi: 10.1016/j.bbih.2020.100144.

11. Chan AO, Huak CY. Psychological impact of the 2003 severe acute respiratory syndrome outbreak on health care workers in a medium size regional general hospital in Singapore. Occup Med (London) 2004;54(3):190-96. doi: 10.1093/ occmed/kqh027.

12. Bai Y, Lin CC, Lin CY, Chen JY, Chue CM, Chou P. Survey of stress reactions among health care workers involved with the SARS outbreak. Psychiatr Serv 2004;55(9):1055-57. doi: 10.1176/ appi.ps.55.9.1055.

13. Wu P, Fang Y, Guan Z, et al. The psychological impact of the SARS epidemic on hospital employees in China: Exposure, risk perception, and altruistic acceptance of risk. Can J Psychiatry 2009;54(5):302-11. doi: 10.1177/070674370905400504.

14. Mo $Y$, Deng $L$, Zhang $L$, et al. Work stress among Chinese nurses to support Wuhan in fighting against COVID-19 epidemic. J Nurs Manag 2020;28(5):1002-09. doi: 10.1111/jonm.13014.

15. Rossi R, Socci V, Pacitti F, et al. Mental health outcomes among frontline and second-line health care workers during the coronavirus disease 2019 (COVID-19) pandemic in Italy. JAMA Netw Open 2020;3(5):e2010185. doi: 10.1001/ jamanetworkopen.2020.10185.

16. García-Fernández L, Romero-Ferreiro V, López-Roldán PD, et al. Mental health impact of COVID-19 pandemic on Spanish healthcare workers. Psychol Med 2020:1-3. doi: 10.1017/ S0033291720002019

17. Liu CY, Yang Y, Zhang XM, et al. The prevalence and influencing factors in anxiety in medical workers fighting COVID-19 in China: A crosssectional survey. Epidemiol Infect 2020;148:e98. doi: 10.1017/S0950268820001107.

18. Zhang WR, Wang K, Yin L, et al. Mental health and psychosocial problems of medical health workers during the COVID-19 epidemic in China. Psychother Psychosom 2020;89(4):242-50. doi: 10.1159/000507639.

19. Lai J, Ma S, Wang Y, et al. Factors associated with mental health outcomes among health care workers exposed to coronavirus disease 2019. JAMA Netw Open 2020;3(3):e203976. doi: 10.1001/jamanetworkopen.2020.3976.

20. Zhang C, Yang L, Liu S, et al. Survey of insomnia and related social psychological factors among medical staff involved in the 2019 novel coronavirus disease outbreak. Front Psychiatry 2020;11:306. doi: 10.3389/fpsyt.2020.00306.

21. Tian T, Meng F, Pan W, et al. Mental health burden of frontline health professionals treating imported patients with COVID-19 in China during the pandemic. Psychol Med 2020:1-2. doi: 10.1017/ S0033291720002093

22. Li G, Miao J, Wang H, et al. Psychological impact on women health workers involved in COVID-19 outbreak in Wuhan: A crosssectional study. J Neurol Neurosurg Psychiatry 2020;91(8):895-97. doi: 10.1136/jnnp-2020323134.

23. Khanna R, Forbes M. Telepsychiatry as a public health imperative: Slowing COVID-19. Aust N Z J Psychiatry 2020;54(7):758. doi: 10.1177/0004867420924480.

24. Zhou $X$, Snoswell CL, Harding LE, et al. The role of telehealth in reducing the mental health burden from COVID-19. Telemed J E Health 2020;26(4):377-79. doi: 10.1089/tmj.2020.0068. 
25. Australian Psychological Society. Stress and Wellbeing: How Australians are coping with life. Melbourne, Vic: APS, 2015.

26. North CS, Ringwalt CL, Downs D, Derzon J, Galvin D. Postdisaster course of alcohol use disorders in systematically studied survivors of 10 disasters. Arch Gen Psychiatry 2011;68(2):173-80. doi: 10.1001/archgenpsychiatry.2010.131.

27. de Goeij MC, Suhrcke M, Toffolutti $V$, van de Mheen D, Schoenmakers TM, Kunst AE. How economic crises affect alcohol consumption and alcohol-related health problems: A realist systematic review. Soc Sci Med 2015;131:131-46. doi: 10.1016/j.socscimed.2015.02.025.

28. Keyes KM, Hatzenbuehler ML, Hasin DS. Stressful life experiences, alcohol consumption, and alcohol use disorders: The epidemiologic evidence for four main types of stressors. Psychopharmacology (Berl) 2011;218(1):1-17. doi: 10.1007/s00213-0112236-1.

29. Ewing JA. Detecting alcoholism: The CAGE questionnaire. JAMA 1984;252(14):1905-07. doi: 10.1001/jama.252.14.1905.

30. World Health Organization, CBM, World Vision International \& UNICEF. Psychological first aid during Ebola virus disease outbreaks (provisional version). Geneva, CH: WHO, 2014.

31. World Health Organization. Mental health and psychosocial considerations during the COVID-19 outbreak. Geneva, CH: WHO, 2020.

32. Gao J, Zheng P, Jia Y, et al. Mental health problems and social media exposure during COVID-19 outbreak. PLoS One 2020;15(4):e0231924. doi: 10.1371/journal.pone.0231924.

33. Bradbury-Jones $C$, Isham L. The pandemic paradox: The consequences of COVID-19 on domestic violence. J Clin Nurs 2020;29(13-14):2047-49. doi: 10.1111/jocn.15296.

34. Ilie PC, Stefanescu S, Smith L. The role of vitamin $D$ in the prevention of coronavirus disease 2019 infection and mortality. Aging Clin Exp Res 2020;32(7):1195-98. doi: 10.1007/ s40520-020-01570-8.

35. NPS MedicineWise. MBS item number changes for vitamin B12, folate and vitamin D tests. Surry Hills, NSW: NPS MedicineWise, 2015.

36. Xiaoming $X$, Ming A, Su H, et al. The psychological status of 8817 hospital workers during COVID-19 epidemic: A cross-sectional study in Chongqing. J Affect Disord 2020;276:555-61. doi: 10.1016/j. jad.2020.07.092

37. Debnath $D$. The dilemma of treating a doctorpatient: A wrestle of heart over mind? Ochsner J 2015;15(2):130-32.

38. Chan SS, Leung GM, Tiwari AF, et al. The impact of work-related risk on nurses during the SARS outbreak in Hong Kong. Fam Community Health 2005;28(3):274-87. doi: 10.1097/00003727200507000-00008.

39. Spitzer RL, Kroenke K, Williams JB, Löwe B. A brief measure for assessing generalized anxiety disorder: The GAD-7. Arch Intern Med 2006;166(10):1092-97. doi: 10.1001/ archinte.166.10.1092.

40. Blevins CA, Weathers FW, Davis MT, Witte TK, Domino JL. The posttraumatic stress disorder checklist for DSM-5 (PCL-5): Development and initial psychometric evaluation. J Trauma Stress 2015;28(6):489-98. doi: 10.1002/jts.22059.

41. Löwe B, Unützer J, Callahan CM, Perkins AJ, Kroenke K. Monitoring depression treatment outcomes with the patient health questionnaire-9. Med Care 2004;42(12):1194-201. doi: 10.1097/00005650-200412000-00006. 